\title{
„Reden ist Silber, Schweigen ist Gold“": Der Fall Kinski und die ärztliche Schweigepflicht
}

\author{
„Speech is Silver, But Silence is Gold“: The Kinski Case and Professional \\ Discretion
}

Autoren

Institute
Helmut Hausner ${ }^{1}$, Hermann Spießl², Göran Hajak ${ }^{1}$

Klinik und Poliklinik für Psychiatrie, Psychosomatik und Psychotherapie der Universität am Bezirksklinikum Regensburg

Klinik für Psychiatrie, Psychotherapie und Psychosomatik, Bezirkskrankenhaus Landshut

\section{Bibliografie}

DOI $10.1055 / \mathrm{s}-0028-1090090$

Psychiat Prax 2009; 36: 2-3

(c) Georg Thieme Verlag KG

Stuttgart · New York.

ISSN 0303-4259

Korrespondenzadresse

Dr. med. Dr. jur.

Helmut Hausner

Bezirksklinikum Regensburg

93042 Regensburg

helmut.hausner@medbo.de
„War Klaus Kinski ein gefährlicher Psychopath?“ Mit dieser reißerischen Fragestellung trug BILD im Juli 2008 Auszüge aus der psychiatrischen Krankenakte des Patienten Kinski in die Öffentlichkeit. Aber auch seriöse überregionale Tageszeitungen ließen sich die Gelegenheit nicht entgehen, die psychiatrische Krankengeschichte eines Prominenten zu sezieren. So berichtete beispielsweise die Frankfurter Allgemeine Zeitung unter der Überschrift „Behandelt in Berlin. Klaus Kinski war kein Psychopath“ interessiert über die psychiatrische Behandlung Kinskis in der Wittenauer Heilstätte, der späteren Karl-BonhoefferNervenklinik in Reinickendorf (Berlin), die heute vom Vivantes-Konzern betrieben wird. Die Berliner Morgenpost sah sich obendrein am 22.Juli 2008 auch zu dem Hinweis veranlasst, dass sich hinter dem jetzt zeitgemäßen Namen der Klinik die frühere „Städtische Irren- und Idioten-Anstalt zu Baldorff" verbirgt. Diese umfangreiche Medienberichterstattung war möglich geworden, nachdem vom Vivantes-Konzern - sicherlich in redlicher wissenschaftlicher Absicht - über 100000 zeithistorische Krankenakten der KarlBonhoeffer-Nervenklinik an das Berliner Landesarchiv übergeben worden waren. Der anfängliche öffentliche Jubel des Landesarchives über den bedeutenden Zuwachs wertvoller historischer Akten verstummte jedoch rasch, als sich zuvorderst nicht die erwarteten Medizinhistoriker, sondern findige Journalisten über den jetzt öffentlich zugänglichen Aktenschatz hermachten und gezielt nach prominenten Einzelschicksalen zu fahnden begannen. Für diese öffentliche Neugier an fremden Krankengeschichten gibt es zahlreiche historische Präzedenzfälle. So werden in der medizinrechtlichen Fachliteratur als Beispiele für postmortale Verletzungen der ärztlichen Schweigepflicht die Veröffentlichung der Krankenakte Bismarcks durch seinen Leibarzt Schwenninger und die Publikation von Bildern des verstorbenen Papstes Pius XII. durch dessen Leibarzt Galeazzi-
Lisi angeführt [1]. Die Hinterbliebenen Klaus Kinskis haben bereits kurz nach der Veröffentlichung seiner Krankengeschichte die Legitimität dieses Vorgehens bezweifelt und wehren sich mit rechtlichen Mitteln gegen den Eingriff in die Persönlichkeitsrechte des Verstorbenen.

Unabhängig davon wie dieser individuelle Rechtsstreit letztlich ausgeht und ob die Sperrfristen des Berliner Landesarchivgesetzes die Freigabe der Kinski-Akten im konkreten Einzelfall erlauben, muss sich die psychiatrische Fachöffentlichkeit jetzt der Frage stellen, wie zukünftig mit dem Archivmaterial unserer Kliniken und Einrichtungen verfahren werden soll. Die ärztliche Schweigepflicht ist eine der traditionellen Säulen eines vertrauensvollen Arzt-PatientenVerhältnisses. Die Verschwiegenheit des Arztes wird bereits im Eid des Hippokrates eingefordert und ist noch heute über die Berufsordnungen der Landesärztekammern Teil des ärztlichen Standesrechts. Auch der Bundesgesetzgeber hat die über den Tod des Patienten hinausreichende Ärztliche Schweigepflicht in $§ 203$ StGB unter Strafandrohung festgeschrieben. Das Bundesverfassungsgericht räumt der Schweigepflicht des Arztes in einer sehr ausführlich begründeten Entscheidung aus dem Jahr 1972 ebenfalls herausragendes Gewicht ein: „Wer sich in ärztliche Behandlung begibt, muss und darf erwarten, dass alles, was der Arzt im Rahmen seiner Berufsausübung über seine gesundheitliche Verfassung erfährt, geheim bleibt und nicht zur Kenntnis Unbefugter gelangt. Nur so kann zwischen Patient und Arzt jenes Vertrauen entstehen, das zu den Grundvoraussetzungen ärztlichen Wirkens zählt, weil es die Chancen der Heilung vergrößert und damit im Ganzen gesehen - der Aufrechterhaltung einer leistungsfähigen Gesundheitsfürsorge dient" [2]. Wer sich in psychiatrische Behandlung begibt, der muss also darauf vertrauen können, dass seine intimsten Lebensgeheimnisse dauerhaft gewahrt und vor einer neugierigen Öffentlichkeit 
geschützt werden. Die Gewährleistung einer ausreichenden Privatsphäre ist psychiatrischen Patienten schon innerhalb des geschützten Lebensbereiches ihrer behandelnden Klinik ein großes Bedürfnis, umso mehr gilt dies gegenüber Außenstehenden [3]. Gerade bei den immer noch hochgradig stigmatisierten psychiatrischen Erkrankungen betreffen öffentliche Vor- und Fehlurteile nicht nur den Patienten selbst, sondern auch seine Nachkommen [4,5]. Zudem enthalten die psychiatrischen Krankengeschichten - speziell bei solchen Erkrankungen die eine familiäre Häufung aufweisen - implizit auch medizinische Informationen über die Nachkommen des Kranken. In kaum einem anderen medizinischen Bereich ist der Patient gezwungen, ähnlich umfangreich seine privaten Lebensumstände, seine intime Gefühls- und Gedankenwelt sowie die höchstpersönlichen Beziehungen zum Partner, zu Angehörigen und zu anderen Bezugspersonen offenzulegen. Der Patient und seine Angehörigen bringen daher den Therapeuten und auch deren Institutionen einen beachtlichen Vertrauensvorschuss entgegen und liefern sich in ihrer personalen Würde und ihrem öffentlichen Ansehen ein Stück weit der dauerhaften Verschwiegenheit und Vertrauenswürdigkeit dieser Institutionen aus. Aus der gesteigerten und auch generationenübergreifenden Schutzbedürftigkeit psychiatrischer Anamnesen und Befunde folgt nach Auffassung der Autoren unmittelbar die Verpflichtung der psychiatrischen Institutionen, dauerhaft den Schutz solcher Patientengeheimnisse sicherzustellen.

Neben den verletzten Persönlichkeitsrechten der Patienten und ihrer Angehörigen gibt es aber noch weitere gewichtige Gründe, leichtsinnige Vorlagen für einen psychiatrischen Sensationsjournalismus mit prominenten Namen zu vermeiden. Das öffentlich verbreitete Bild von „Kinski dem Irren“ vermischt eine tatsächliche persönliche Leidensgeschichte mit der fiktiven Wahrnehmung einer bekannten Schauspielerpersönlichkeit, wie sie jeder aufgrund seiner Rollen zu kennen meint. Den vielgestaltigen Be- mühungen um eine Entstigmatisierung psychischer Erkrankungen fügen solche Formen der Öffentlichkeitsarbeit schweren Schaden zu [6-8].

Der Fall Kinski hat gezeigt, dass offenbar die Übergabe von Krankenakten an öffentliche Archive mit einem erheblichen Risiko für den Schutz von Patientengeheimnissen und den berechtigten Interessen der Nachkommen psychisch Kranker verbunden sein kann. Daher sollten zur Gewährleistung einer generationenübergreifenden ärztlichen Vertraulichkeit die historischen Krankenaktenbestände psychiatrischer Kliniken möglichst dauerhaft im geschützten Archiv der jeweiligen Einrichtung verbleiben, um Vertrauensbrüche, wie den gegenüber der Familie von Klaus Kinski, zukünftig zu verhindern.

\section{Literatur}

1 Deutsch E, Spickhoff A. Medizinrecht. Berlin, Heidelberg: Springer, 2008

2 BVerfG. Beschlagnahme der ärztlichen Karteikarte eines Beschuldigten. Beschluss vom 8.3.1972. NJW 1972; 25: 1123-1126

3 Spießl H, Kovatsits U, Frick $U$ et al. Privatsphäre auf psychiatrischen Stationen. Psychiat Prax 2002; 29: 10-13

4 Schmid R, Spießl H, Cording C. Zwischen Verantwortung und Abgrenzung: Emotionale Belastungen von Angehörigen psychisch Kranker. Psychiat Prax 2005; 32: 272-280

5 Schmid R, Schielein T, Spießl H et al. Belastungen von Geschwistern schizophrener Patienten. Psychiat Prax 2006; 33: 177-183

6 Holzinger A, Dietrich S, Heitmann S et al. Evaluation zielgruppenorientierter Interventionen zur Reduzierung des Stigmas psychischer Krankheit. Eine systematische Übersicht. Psychiat Prax 2008 DOI: 10.1055/s-2008-1067396

7 Wundsam K, Pitschel-Walz G, Leucht S et al. Psychisch Erkrankte und Angehörige unterrichten Polizeibeamte: Ein Anti-Stigma-Projekt von „BASTA - dem Bündnis für psychisch erkrankte Menschen“. Psychiat Prax 2007; 34: 181-187

8 Rommel C. Psychiatrie backstage - Ein Projektbericht zu Transparenz und Entstigmatisierung der Krankenhauspsychiatrie. Psychiat Prax 2007; 34: 406-407 